\title{
Strategies for pediatric lung transplantation: bridging, listing and surgical technique
}

\author{
Daniel Rinewalt ${ }^{1}$, Sylvia M. Cruz ${ }^{2}$, Michael Wong ${ }^{2}$, Francis Fynn-Thompson ${ }^{3}$, Steve Singh ${ }^{4}$ \\ ${ }^{1}$ Division of Cardiac Surgery, Brigham and Women's Hospital, Boston, MA, USA; ${ }^{2}$ Department of Cardiothoracic Surgery, Stanford University, Palo Alto, \\ CA, USA; ${ }^{3}$ Department of Cardiac Surgery, Boston Children's Hospital, Boston, MA, USA; ${ }^{4}$ Trillium Health Partners, Mississauga, Ontario, Canada \\ Correspondence to: Daniel Rinewalt, MD. Division of Cardiac Surgery, Brigham and Women's Hospital, 75 Francis Street, Boston, MA 02115, USA. \\ Email: drinewalt@partners.org.
}

Submitted Sep 02, 2019. Accepted for publication Oct 30, 2019.

doi: 10.21037/acs.2019.11.01

View this article at: http://dx.doi.org/10.21037/acs.2019.11.01

\section{Introduction}

Lung transplantation remains a valuable treatment modality for end-stage lung disease in the pediatric population, despite being relatively uncommon when compared to the adult population. There were 35 pediatric lung transplants in the United States in 2018, with only 10 of these taking place in patients under the age of 11 (1). The Scientific Registry of Transplant Recipients (SRTR) and the Organ Procurement and Transplantation Network (OPTN) released the last joint annual report in 2017, revealing that 30 patients were added to the waitlist that year with the majority (59.1\%) aged 6-11 (2). Of patients on the waitlist for $2017,59 \%$ underwent lung transplantation while $30 \%$ died awaiting a suitable organ (2). Survival for pediatric lung transplantation reported for $2005-2012$ is $83.2 \%$ at one year, $66.4 \%$ at three years, and $59.7 \%$ at five years (2). Predictably, the most common cause of mortality at five years is graft failure present in $9.7 \%$, with evidence of chronic rejection in the form of bronchiolitis obliterans, present in $35 \%$ (2). Of patients surviving to five years, $91 \%$ are reported as fully active, indicating the possibility of an excellent quality of life in this population after transplantation (2).

\section{Listing}

There are currently two distinct systems which prioritize pediatric patients for lung transplantation. If over the age of 12 , patients are listed in order of the lung allocation score (LAS), similar to adults (3). For younger patients and infants, there is a two-tiered system based on perceived medical urgency denoted as Priority 1 and Priority 2 (3). In order to be assigned the highest priority (1), the patient must have respiratory failure or pulmonary hypertension by criteria that are strictly defined by the OPTN (3). Cystic fibrosis remains the most common diagnosis leading to lung transplantation in the pediatric population overall, although for infants, congenital disease remains more common (4).

The challenge in listing pediatric patients for transplantation will always be in finding a balance between the life expectancy of the disease process itself, which is often times unpredictable, with that of post-transplantation survival. While many centers will refer patients for lung transplantation when life expectancy is thought to be less than two years, it is not uncommon for pediatric patients to wait longer for a suitable organ when compared to adults. Interestingly, with the implementation of the LAS score, there has been an improvement in waitlist time and mortality, including in the pediatric population; however, the effect has not been as significant when compared to adults (5). As the LAS score does not include any models of pediatric disease, it likely does not capture the degree of critical illness in this population (5). In order to further eliminate discrepancies in waitlist times, exceptions have been granted to pediatric patients to participate in LAS scoring in selected cases, in addition to changes in organ allocation policy to allow for greater geographic sharing of pediatric organs (4). 


\section{Bridging}

The majority of critically ill pediatric patients requiring a bridge to lung transplantation are done so with mechanical ventilation. While extracorporeal membrane oxygenation (ECMO) has been successfully described in the pediatric population, it is not as commonplace when compared to the adult experience in lung transplantation. As the singlesite insertion double-lumen catheters are often times too large for most patients, dual site cannulation (i.e., femoral and internal jugular) is required, which limits ambulation. Despite reports of worse outcomes when bridging to transplantation with ECMO in pediatrics, Hayes reported 17 patients successfully transitioned from ECMO to lung transplantation with acceptable survival (6).

\section{Surgical technique}

Surgical technique in the pediatric population does not drastically differ from that in adults, with the exception that the majority of these operations are bilateral and take place on cardiopulmonary bypass (CPB). It is uncommon for double-lumen tubes to be small enough to accommodate the pediatric population. Additionally, patients with pulmonary hypertension and congenital heart disease may not tolerate single-lung ventilation and perfusion or require additional procedures at the time of transplantation. A clamshell thoracotomy provides adequate exposure to the hilar structures; however, a median sternotomy is also acceptable and is certainly our preferred technique in adults. We cannulate the aorta and right atrium centrally and do very little hilar dissection without CPB: it is not our practice to arrest the heart. We perform bilateral pneumonectomies by stapling across the pulmonary artery and pulmonary veins, then divide the bronchus sharply in order to suction out the contents before the donor lungs are brought into the field. We perform the bronchial anastomosis with a running 4-0 PDS on the membranous (back wall) surface, then complete the cartilaginous portion (front wall) using interrupted 4-0 Vicryl sutures.

The pulmonary artery anastomosis is performed next, with the use of a side-biting vascular clamp using a running $5-0$ prolene, followed by the pulmonary vein anastomosis with a running 4-0 prolene (without tying at anastomosis completion), also with the use of a vascular clamp. We eliminate intracardiac air by releasing the pulmonary artery clamp, asking our perfusionist to leave volume in the heart, releasing the pulmonary venous clamp, and finally tying the pulmonary vein suture. We then repeat for the opposite lung and re-perfuse for approximately 15 minutes before weaning off $\mathrm{CPB}$.

\section{Conclusions}

Lung transplantation is the gold standard for end-stage lung disease in the pediatric population, but it is currently limited to few centers, given the low volume of procedures overall. Continued improvement in prognostication and expanded use of donor organs will likely be the focus of future research endeavors in order to yield decreased waitlist times and mortality.

\section{Acknowledgments}

None.

\section{Footnote}

Conflicts of Interest: The authors have no conflicts of interest to declare.

\section{References}

1. Organ Procurement and Transplantation Network (US) [Online]. 2019. Transplants in the U.S. by Recipient Age. Available online: https://optn.transplant.hrsa.gov/data/ view-data-reports/national-data/

2. 2017 Annual Report of the U.S. Organ Procurement and Transplantation Network and the Scientific Registry of Transplant Recipients: Lung. Department of Health and Human Services, Health Resources and Services Administration, Healthcare Systems Bureau, Division of Transplantation, Rockville, MD; United Network for Organ Sharing, Richmond, VA; University Renal Research and Education Association, Ann Arbor, MI. Available online: https://srtr.transplant.hrsa.gov/annual_ reports/2017/Lung.aspx

3. Organ Procurement and Transplantation Network (US) [Online]. 2019. Organ Procurement and Transplantation Network Policies. Available online: https://optn.transplant. hrsa.gov/media/1200/optn_policies.pdf

4. Lancaster TS, Eghtesady P. State of the Art in Pediatric Lung Transplantation. Semin Thorac Cardiovasc Surg 2018;30:166-74.

5. Lancaster TS, Miller JR, Epstein DJ, et al. Improved waitlist and transplant outcomes for pediatric lung 
transplantation after implementation of the lung allocation score. J Heart Lung Transplant 2017;36:520-8.

6. Hayes D, Mcconnell PI, Tobias JD, et al. Survival in children on extracorporeal membrane oxygenation at the time of lung transplantation. Pediatr Transplant 2015;19:87-93.
Cite this article as: Rinewalt D, Cruz SM, Wong M, Fynn-Thompson F, Singh S. Strategies for pediatric lung transplantation: bridging, listing and surgical technique. Ann Cardiothorac Surg 2020;9(1):51-53. doi: 10.21037/ acs.2019.11.01 